\title{
OPTIMIZATION OF FRAMES WITH SEMI-RIGID CONNECTIONS
}

\author{
L. M. C. Simōes \\ Departamento de Engenharia Civil, Faculdade de Ciências e Tecnologia Universidade de Coimbra, \\ 3049 Coimbra Codex, Portugal
}

(Received 29 April 1995)

\begin{abstract}
This work describes a computer-based method for the optimum design of steel frameworks accounting for the behaviour of semi-rigid connections. The procedure explicitly accounts for both connections and members by taking connection stiffnesses and member sizes as continuous-valued and discrete-valued design variables, respectively. The optimization algorithm minimizes the cost of the connections and members of the structure subjected to constraints on stresses and displacements under specified design loads. Two examples are presented to illustrate the features of the optimization method. Copyright (C) 1996 Elsevier Science Ltd.
\end{abstract}

\section{INTRODUCTION}

Traditional approaches to steel frame design neglect the actual behaviour of connections. Instead, two idealizations are used: pinned and fully rigid. Although these models simplify analysis and design procedures, the predicted response of the frame may not be realistic. In practice most connections transmit some moments and experience some rotations that can contribute substantially to overall structure displacements. The term semi-rigid is commonly used to denote the connection behaviour between these two extremes. When a beam-column assembly is tested. for a given moment, a corresponding rotation is obtained for the beam plus connection. If the elastic rotation of the beam is subtracted, the applied moment may be plotted against the characteristic rotation of the connection. This is referred as the $M / \phi$ curve [1-3]. A selection of $M / \phi$ curves is represented in Fig. 1. It is apparent that the behaviour is nonlinear, as an initial stiff phase is followed by a second phase of much reduced stiffness and the shape of the curve depends on the exact form of connection. Flexible connection behaviour affects the internal force distribution in the members of a framework. By treating the connection as semirigid a more reliable prediction of frame behaviour is obtained. Additional economy can be achieved by making use of the stiffness and strength of connections that would otherwise be treated as pinned and also by avoiding the stiffening often required in rigid connections.

In the past three decades, considerable research has been carried out to assess the actual behaviour of steel frameworks accounting for the effect of connection fexibility. Although a semi-rigid connection framework design has been adopted by Eurocode 3 and America Institute of Steel Construction specifications, specific guidelines for the design of such frame- works are not provided or readily available. Current research interest in semi-rigid design in Europe and elsewhere is extensive. Research is ongoing and it is hoped that this will result in sufficient information being available to allow engineers full use of this method in the future. However, much of this research has been limited to the analysis problem, with little attention being paid to the important problem of optimization [4]. This work presents a systematic method for the optimum design of steel frameworks accounting for the behaviour of semi-rigid connections. The design which has the minimum combined cost of members and connections is sought, while also ensuring that stresses and displacements are within acceptable limits. Members are sized using discrete standard steel sections, while connections are selected on the basis of their continuous-valued moment rotation stiffnesses. The segmental method [6-7] which uses linear programming is adopted for optimization purposes. The sensitivity analysis is carried out by analytic means. Two steel frameworks examples are presented to illustrate the features of this design method.

\section{ANALYSIS OF FRAMEWORKS WITH SEMI-RIGID CONNECTIONS}

Sophisticated methods of frame analysis are already available. These enable all significant influences such as the nonlinearities resulting from material behaviour and the geometry of the structure. The connection is usually represented by fictitious structural elements at the ends of members. These elements are assigned predetermined relationship between forces and displacements so as to simulate the behaviour of the joint as a whole. The most simple model to analyse frames with semi-rigid connections is a linear representation of the spring which in many cases will be quite adequate. The effects of connection flexibility are 


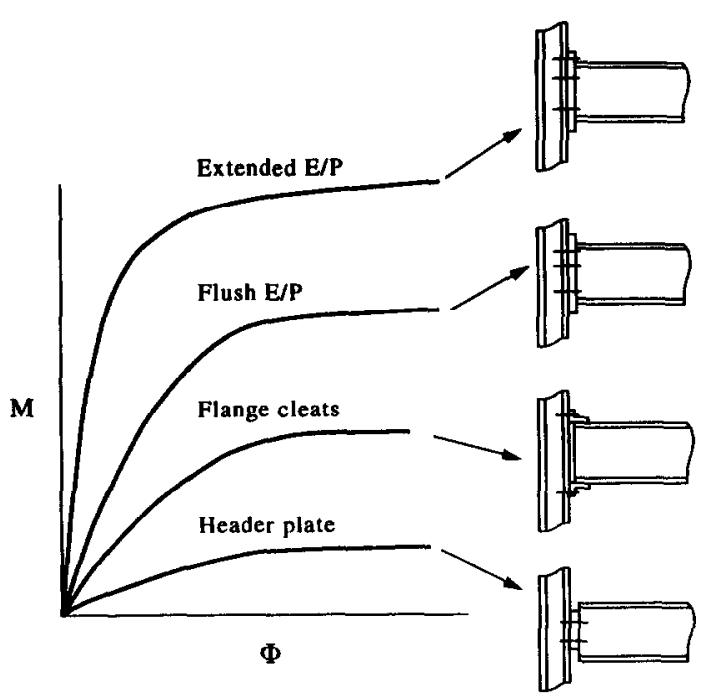

Fig. 1. $M / \phi$ comparisons for various connection types.

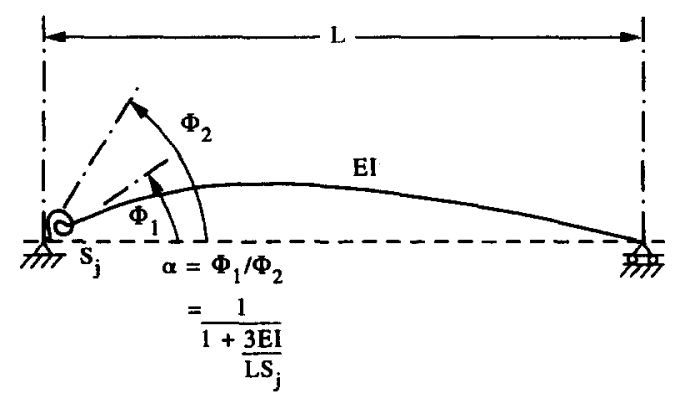

Fig. 2. Fixity factor.

modelled by attaching rotational springs of moduli $S_{1}$ and $S_{2}$ normally measured in $\mathrm{kNm} \mathrm{rad}{ }^{-1}$ to the ends 1 and 2 of a member, as shown in Fig. 2.

According to a first-order analysis, the stiffness matrix of a planar member with semi-rigid restraint at the ends can be represented by the stiffness matrix $K_{i}$ for the member with rigid connections modified by a correction matrix [5]:

$$
\left[\begin{array}{cccccc}
\frac{E A}{L} & 0 & 0 & -\frac{E A}{L} & 0 & 0 \\
0 & \frac{12 E I}{L^{3}} \frac{\left(\alpha_{1}+\alpha_{2}+\alpha_{1} \alpha_{2}\right)}{\left(4-\alpha_{1} \alpha_{2}\right)} & \frac{6 E I}{L^{2}} \frac{\left(2 \alpha_{1}+\alpha_{1} \alpha_{2}\right)}{\left(4-\alpha_{1} \alpha_{2}\right)} & 0 & \frac{12 E I}{L^{3}} \frac{\left(\alpha_{1}+\alpha_{2}+\alpha_{1} \alpha_{2}\right)}{\left(4-\alpha_{1} \alpha_{2}\right)} & \frac{6 E I\left(2 \alpha_{2}+\alpha_{1} \alpha_{2}\right)}{L^{3}} \frac{\left(4-\alpha_{1} \alpha_{2}\right)}{0} \\
0 & \frac{6 E I}{L^{2}} \frac{\left(2 \alpha_{1}+\alpha_{1} \alpha_{2}\right)}{\left(4-\alpha_{1} \alpha_{2}\right)} & \frac{4 E I}{L} \frac{\left(3 \alpha_{1}\right)}{\left(4-\alpha_{1} \alpha_{2}\right)} & 0 & -\frac{6 E I}{L^{2}} \frac{\left(2 \alpha_{1}+\alpha_{1} \alpha_{2}\right)}{\left(4-\alpha_{1} \alpha_{2}\right)} & \frac{2 E I}{L} \frac{\left(3 \alpha_{1} \alpha_{2}\right)}{\left(4-\alpha_{1} \alpha_{2}\right)} \\
-\frac{E A}{L} & 0 & 0 & \frac{E A}{L} & 0 & 0 \\
0 & \frac{12 E I}{L^{3}} \frac{\left(\alpha_{1}+\alpha_{2}+\alpha_{1} \alpha_{2}\right)}{\left(4-\alpha_{1} \alpha_{2}\right)} & -\frac{6 E I}{L^{2}} \frac{\left(2 \alpha_{1}+\alpha_{1} \alpha_{2}\right)}{\left(4-\alpha_{1} \alpha_{2}\right)} & 0 & \frac{12 E I}{L^{3}} \frac{\left(\alpha_{1}+\alpha_{2}+\alpha_{1} \alpha_{2}\right)}{\left(4-\alpha_{1} \alpha_{2}\right)} & -\frac{6 E I}{L^{3}} \frac{\left(2 \alpha_{2}+\alpha_{1} \alpha_{2}\right)}{\left(4-\alpha_{1} \alpha_{2}\right)} \\
0 & \frac{6 E I}{L^{2}} \frac{\left(2 \alpha_{2}+\alpha_{1} \alpha_{2}\right)}{\left(4-\alpha_{1} \alpha_{2}\right)} & \frac{2 E I}{L} \frac{\left(3 \alpha_{1} \alpha_{2}\right)}{\left(4-\alpha_{1} \alpha_{2}\right)} & 0 & -\frac{6 E I}{L^{2}} \frac{\left(2 \alpha_{2}+\alpha_{1} \alpha_{2}\right)}{\left(4-\alpha_{1} \alpha_{2}\right)} & \frac{4 E I}{L} \frac{\left(3 \alpha_{2}\right)}{\left(4-\alpha_{1} \alpha_{2}\right)}
\end{array}\right]
$$


most powerful and important concept for the analysis of frames with semi rigid joints. It relates quite closely to how the structure will behave in the context of the connection, far more so than the absolute value of $S_{j}$. Figure 3 indicates a typical variation in $S_{j}$ against $\alpha_{j}$.

As it can be seen, a real pin connection will always have some stiffness and will lead to significant restraint moments which could be of great benefit to a structure. Also, large reductions in stiffness from full fixity are seen to have little effect on the structure in that only a small change in $\alpha$ will result in a very large physical change to $S_{j}$. The fact that $\alpha_{j}$ smooths the variations in $S_{j}$ confirms that only an approximately accurate $M / \phi$ curve is required, as the analysis is directly affected by $x$ and not $S_{j}$.

In the linear elastic analysis, the connection and members are assumed to have linear force-displacement relationships and the effect of deformation on the equilibrium of the frame is disregarded. In its usual form, the analysis requires the solution of the set of linear equations:

$$
\mathbf{K} u=P .
$$

The advantage of this approach is that the overall procedure is the same as the conventional matrix displacement method commonly adopted for rigidjointed frames. The end-moments for a semi-rigidly connected planar member of length $L$ under uniformly distributed loading $p$ are:

$$
\begin{aligned}
& M_{1}=\frac{p L^{2}}{12} \frac{3 \alpha_{1}\left(2-\alpha_{2}\right)}{\left(4-\alpha_{1} \alpha_{2}\right)} ; \\
& M_{2}=-\frac{p L^{2}}{12} \frac{3 \alpha_{2}\left(2-\alpha_{1}\right)}{\left(4-\alpha_{1} \alpha_{2}\right)} .
\end{aligned}
$$

\section{STRUCTURAL OPTIMIZATION}

The optimization problem consists of finding the minimum combined cost of members and connections while accounting for the semi-rigid behaviour of the connections. The connection behaviour, as reflected through the fixity factor, has a significant influence on the response of the structure and, as such, will also have considerable effect on the cost of the structure. Herein, the cost of each member $i$ is represented by its weight, while the cost of each connection $k$ is taken to be directly related to the fixity factor. Therefore, the total cost of a member $i$ with two end-connections $k=1,2$ may be expressed as

$$
Z_{i}=w_{i} a_{i}+\Sigma_{k-1.2}\left(V_{i k}^{0}+V_{i k}^{1} \alpha_{i k}+V_{i k}^{2} \alpha_{i k}^{2}\right),
$$

where $a_{i}$ and $w_{i}$ are the member cross-section area and weight coefficient $\left(w_{i}=\rho_{i} l_{i}\right.$, i.e. material density $\times$ member length), $\alpha_{i k}, V_{i k}^{1}$ and $V_{i k}^{2}$ are the fixity factor and cost coefficients and $V_{i k}^{0}$ is the cost of a pinned connection having zero rotational stiffness.
Although there is little or no information in the literature concerning the value of the cost coefficients for the various types of connections used in steel frameworks, representative values for $V_{i k}^{1}$ and $V_{i k}^{2}$ will be derived in the following section.

Each member $i$ is to be sized using a commercial standard steel section and, as such, its cross-section area $a_{i}$ is a discrete design variable. On the other hand, each connection $k$ may be fabricated anywhere in the range from being fully-pinned to fully-fixed and, as such, its fixity factor $\alpha_{i k}$ is a continuous design variable.

$$
a_{i} \in A_{i} ; \quad 0 \leqslant \alpha_{i k} \leqslant \mathrm{I},
$$

where eqn (6a) requires the cross-section properties of member $i$ to belong to the discrete set $A_{i}=\left\{a_{1}, a_{2}, \ldots\right\}$ prevailing for the standard section shape specified (e.g. IPE shape), while eqn (6b) imposes specified lower and upper bounds.

The optimal design of a framework of $i=1,2$, $\ldots, n$ members having semi-rigid connections subject to stress and displacement constraints, may be generally stated as,

$$
\begin{aligned}
\operatorname{Min} & Z=\Sigma_{i=1, n} \\
\times & {\left[w_{i} a_{i}+\Sigma_{k=1.2}\left(V_{i k}^{0}+V_{i k}^{1} \alpha_{i k}+V_{i k}^{2} \alpha_{i k}^{2}\right)\right], }
\end{aligned}
$$

subject to:

$$
\begin{aligned}
& \sigma_{i}^{L} \leqslant \sigma_{i} \leqslant \sigma_{i}^{l^{\prime}}, \\
& u_{1}^{L} \leqslant u_{1} \leqslant u_{1}^{L}, \\
& 0 \leqslant \alpha_{i k} \leqslant 1, \\
& a_{i} \in A_{i},
\end{aligned}
$$

where, from eqn (5), the objective function eqn (7a) is a measure of the combined cost of the members and connections. Equations (7b) and (7c) define constraints on stresses $\sigma_{k}$ and displacements $u_{1}$ for the structure and, from eqn (6), the constraints eqns (7d) and (7e) control the values of the continuous-valued fixity factors $\alpha_{i j}$ and the discrete-valued member sizes $a_{i}$, respectively.

\subsection{Connection cost coefficients}

Published data suggests that the cost of a steel member with IPE section is increased by $20 \%$ if it has pin-jointed end-connections, and by $60 \%$ if its end-connections are bolted or welded. Therefore, this means the total member plus connection cost defined by eqn (5) is such that

$$
\begin{aligned}
1.2 w_{i} a_{i} & \leqslant w_{i} a_{i}+\Sigma_{k=1.2}\left(V_{i k}^{0}+V_{i k}^{1} \alpha_{i k}+V_{i k}^{2} \alpha_{i k}^{2}\right) \\
& \leqslant 1.6 w_{i} a_{i} .
\end{aligned}
$$


Recognizing that the cost of a member with pin-jointed connections is

$$
w_{i} a_{i}+\Sigma_{k=1,2} V_{i k}^{0}=1.2 w_{i} a_{i},
$$

and the fact that $V_{i 1}^{0}=V_{i 2}^{0}$, it follows from eqn (9) that the cost of a pin-jointed connection is $V_{i k}^{0}=$ $0.1 w_{i} a_{i}$. If a linear variation of cost between a pinned and a fully rigid connection is assumed, $V_{i 1}^{2}=V_{i 2}^{2}=0$ and $V_{i 1}^{1}=V_{i 2}^{1}=0.2 w_{i} a_{i}$.

$\Lambda$ more accurate connection cost is obtained by considering a nonzero coefficient for the quadratic term in $\alpha_{i k}$, reflecting the increase in cost to provide either a perfectly fixed or perfectly pinned connection. Average connection cost coefficients are given by:

$$
\begin{aligned}
& V_{i k}^{0}=0.1 w_{i} a_{i}, \quad V_{i 1}^{1}=V_{i 2}^{1}=-0.4 w_{i} a_{i} ; \\
& V_{11}^{2}=V_{i 2}^{2}=0.6 w_{i} a_{i},
\end{aligned}
$$

and the total (material plus connection) cost of a member with semi-rigid joints in eqn (7a) becomes a nonlinear function coupling the design variables $a_{i}$ and $\alpha_{i}$ :

$$
w_{i} a_{i}+0.2 w_{i} a_{i}-0.8 w_{i} a_{i} \alpha_{i}+1.6 w_{i} a_{i} \alpha_{i}^{2}
$$

\subsection{Explicit approximation problem}

To enable a computer solution of the design optimization problem posed by eqn (7), it is first necessary to formulate each stress $\sigma_{k}$ and displacement $u_{1}$ in eqn (7b) and (7c) as an explicit function of the design variables. Since stresses and displacements in frame structure vary inversely with the section properties (area, bending moment of inertia) and directly with fixity factors, a good quality explicit approximation of each stress $\sigma_{j}$ is provided by the first-order Taylor series:

$$
\begin{aligned}
\sigma_{j}= & \sigma_{0 j}+\sum_{i=1}^{n}\left\{\frac{\partial \sigma_{0 j}}{\partial \mathrm{l} / a_{i}}\left[\frac{1}{a_{i}}-\frac{1}{a_{0 i}}\right]+\frac{\partial \sigma_{0 j}}{\partial 1 / I_{i}}\left[\frac{1}{I_{i}}-\frac{1}{I_{0 i}}\right]\right\} \\
& +\sum_{i=1}^{n} \frac{\partial \sigma_{0 j}}{\partial x_{i}}\left(\alpha_{i}-\alpha_{0 i}\right)
\end{aligned}
$$

while that for each displacement $u_{i}$ is:

$$
\begin{aligned}
u_{1}= & u_{01}+\sum_{i=1}^{n}\left\{\frac{\partial u_{01}}{\partial \mathrm{l} / a_{i}}\left[\frac{1}{a_{i}}-\frac{1}{a_{0 i}}\right]+\frac{\partial u_{01}}{\partial \mathrm{l} / I_{i}}\left[\frac{1}{I_{i}}-\frac{1}{I_{0 i}}\right]\right\} \\
& +\sum_{i=1}^{n} \partial u_{01} \partial \sigma_{i}\left(\alpha_{i}-\alpha_{0 i}\right),
\end{aligned}
$$

where the superscript zero $(0)$ defines known quantities for the current structure, while $a_{i}\left(\right.$ or $\left.I_{i}\right)$ and $\alpha_{i}$ are the variables to the design.

Assuming that costs per unit volume are constant, the material cost of the steel frame is a linear function of the sizing design variables and does not need to be considered further. The nonlinear connection cost function is known explicitly and the derivatives can be obtained by direct differentiation of eqn (11). The first order Taylor series approximation gives:

$$
\begin{aligned}
& 1.2 w_{i} a_{i}-0.8 w_{i}\left(a_{0 i} \alpha_{i}+a_{i} \alpha_{0 i}-a_{0 i} \alpha_{0 i}\right) \\
& +1.6 w_{i}\left(a_{i} \alpha_{0 i}^{2}+2 a_{0 i} \alpha_{0 i} \alpha_{i}-2 a_{0 i} \alpha_{0 i}^{2}\right) \\
= & w_{i} a_{i}+w_{i}\left(c_{i}^{0}+c_{i}^{1} a_{i}+c_{i}^{2} \alpha_{i}\right) .
\end{aligned}
$$

Nlthough the fixity factors may vary between the perfectly pinned and the fully fixed cases, move limits should be imposed on $\alpha_{j}$ to reduce inaccuracies associated with the computation of the explicit approximations on the stresses and displacements. Therefore the optimation problem expressed explicitly in terms of the design variables is:

$$
\operatorname{Min} Z-\sum_{i=1 . n}\left[w_{i} a_{i}+w_{i}^{1}\left(c_{i}^{0}+c_{i}^{1} a_{i}+c_{i}^{2} \alpha_{l}\right)\right],
$$

subject to

$$
\begin{aligned}
& u_{1}^{L} \leqslant u_{01}+\sum_{i=1}^{n}\left\{\frac{\partial u_{01}}{\partial 1 / a_{i}}\left[\frac{1}{a_{i}}-\frac{1}{a_{0 i}}\right]\right. \\
& \left.+\frac{\partial u_{01}}{\partial 1 / I_{i}}\left[\frac{1}{I_{i}}-\frac{1}{I_{0 i}}\right]\right\} \\
& +\sum_{i=1}^{n} \frac{\partial u_{01}}{\partial \alpha_{i}}\left(\alpha_{i}-\alpha_{0 i}\right) \leqslant u_{1}^{U}, \\
& \sigma_{j}^{L} \leqslant \sigma_{0 j}+\sum_{i=1}^{n}\left\{\frac{\partial \sigma_{0 j}}{\partial 1 / a_{i}}\left[\frac{1}{a_{i}}-\frac{1}{a_{0 i}}\right]\right. \\
& \left.+\frac{\partial \sigma_{0 j}}{\partial 1 / I_{i}}\left[\frac{1}{I_{i}}-\frac{1}{I_{0 i}}\right]\right\} \\
& +\sum_{i=1}^{n} \frac{\partial \sigma_{0 j}}{\partial \alpha_{i}}\left(\alpha_{i}-\alpha_{0 i}\right) \leqslant \sigma_{j}^{U}, \\
& \alpha_{i}^{L} \leqslant \alpha_{i} \leqslant \alpha_{i}^{U}, \\
& a_{i} \in A_{i} .
\end{aligned}
$$

The continuous-discrete optimization problem, eqn (14), is solved at each design stage to find new values of $a_{i}$ (or $I_{i}$ ) and $\alpha_{j}$ for which the combined member plus connection cost of the structure is reduced relative to that for the previous design stage. The stress and displacement gradients are then updated for this new structure and the design optimization is conducted again. This process is repeated until cost convergence occurs for successive design stages, at which point the minimum cost design has been found. Note again that the design optimization problem involves both continuous-valued variables $\alpha_{i}$ and discrete-valued variables $a_{i}$ (or $I_{i}$ ).

\section{SENSITIVITY ANALYSIS}

The derivatives of the stresses and displacements with respect to the design variables are found by the 
virtual load procedure. Given some design variable values a full analysis of the frame will give numerical values for all the nodal displacement under all loading cases. The displacement derivatives $\partial u / \partial 1 / a_{i}$ and $\partial u / \partial 1 / I_{i}$ are computed by implicit differentiation of the equilibrium equations:

$$
\mathbf{K}_{0} \frac{\partial u_{0}}{\partial 1 / a_{i}}=-\frac{\partial K_{0}}{\partial 1 / a_{i}} u_{0} ; \quad \mathbf{K}_{0} \frac{\partial u_{0}}{\partial 1 / I_{i}}=-\frac{\partial K_{0}}{\partial 1 / I_{i}} u_{0} .
$$

Since $u$ and $K$ arc known from the analysis of the initial design, a solution for $\partial u / \partial 1 / a_{i}$ and $\partial u / \partial 1 / I_{i}$ involves only calculation of the r.h.s. vector of eqn (15) and forward and back substitutions. To compute $\partial \mathbf{K} / \partial x_{i}$, only elements of $\mathbf{K}$ associated with member $i$ must be considered and all are constants. Assuming that $\mathbf{R}_{n}$ and $\mathbf{R}_{m}$ are transformation matrices giving the axial and bending components of the normal stress, respectively:

$$
\begin{gathered}
\sigma=\sigma_{n}+\sigma_{m}=\mathbf{R}_{n} u+\mathbf{R}_{m} u \\
\frac{\partial \sigma_{0}}{\partial \mathbf{l} / a_{i}}=\mathbf{R}_{n} \frac{\partial u_{0}}{\partial \mathbf{1} / a_{i}} ; \quad \frac{\partial \sigma_{0}}{\partial 1 / I_{i}}=\mathbf{R}_{m} \frac{\partial u_{0}}{\partial 1 / I_{i}} .
\end{gathered}
$$

The derivatives $\partial u / \partial \alpha_{i}$ and $\partial \sigma / \partial \alpha_{i}$ are computed in a similar way. However, it should be remembered that the $\partial P / \partial \alpha_{i}$ terms must be considered:

$$
\mathbf{K}_{0} \frac{\partial u_{0}}{\partial \alpha_{i}}=\frac{\partial P_{0}}{\partial \alpha_{i}}-\frac{\partial \mathbf{K}_{0}}{\partial \alpha_{i}} u_{0},
$$

and since

$$
\sigma=Q u,
$$

the stress gradients are

$$
\frac{\partial \sigma_{0}}{\partial \alpha_{i}}=Q_{0} \frac{\partial u_{0}}{\partial \alpha_{i}} .
$$

\section{SEGMENTAL OPTIMUM DESIGN}

An algorithm for the direct solution of problem (14) must include provisions to overcome the stress and displacement constraints non-linearly and the discreteness requirement stated by eqn (14e). The rigorous discrete-continuous optimum design is a NP hard problem, significantly more difficult than the con- tinuous problem. The continuous optimum design forms a lower bound to the discrete optimum and it is usually assumed that the continuous sizes should somehow be rounded up or down to discrete sizes. This rounding process turns out also to be a combinatorial problem. The method described next introduces the artificial concepts of segmental members and segmental optimum design and provides a close lower bound to the discrete-continuous optimum problem.

Problem (14) assumes that each member is of known length $L_{i}$ and has unknown, but uniform crosssectional properties (area $A_{i}$, modulus $W_{i}$, inertia $I_{i}$ ). Consider replacing this assumption by a different one. Assume instead that each member of the frame is composed of a total of $D$ segments, each with a crosssectional area equal to one of the discrete sizes $S_{d}$, $d=1, \ldots, D$, such that all sizes are represented among the segments. Let $l_{i d}$ be the unknown length of the segment of member $i$ which has area $S_{d}, d=1$, $\ldots, D$. This is shown in Fig. 4 for a member which has three discrete sizes. The areas of all segments are known, but the segment lengths are unknown. The ordering of segments along a member is immaterial. Clearly, to replace a conventional member by a segmental one their total lengths must be identical. Thus for member $i$, relationship (21) must hold:

$$
\Sigma_{d=1 . D} l_{i d}=L_{i} ; \quad i=1, \ldots, N .
$$

If problem (14) is re-formulated with this segmental assumption in place of the uniform members the following problem is obtained:

$$
\begin{aligned}
\operatorname{Min} Z= & \Sigma_{i=1, n} \Sigma_{d=1 . D}\left[\rho_{i} s_{d} l_{i d}\right. \\
& \left.+\rho_{i}\left(c_{i}^{0} l_{i}+c_{i}^{1} s_{d} l_{i d}+c_{i}^{2} l_{i} \alpha_{i}\right)\right]
\end{aligned}
$$

subject to

$$
\begin{aligned}
& u_{1}^{L} \leqslant u_{0 l}+\sum_{i=1}^{n}\left\{\frac{\partial u_{0 t}}{\partial 1 / a_{i}}\left[\sum_{d-1}^{D} \frac{1}{s_{d}} \frac{l_{i d}}{l_{i}}-\frac{1}{a_{0 i}}\right]\right. \\
& +\frac{\partial u_{0 l}}{\partial 1 / I_{i}}\left[\sum_{d=1}^{D} \frac{1}{I_{d}} \frac{l_{i d}}{l_{i}}-\frac{1}{I_{0 i}}\right] \\
& \left.+\frac{\partial u_{0 t}}{\partial \alpha_{i}}\left(\alpha_{i}-\alpha_{0 i}\right)\right\} \leqslant u_{i}^{\nu}
\end{aligned}
$$
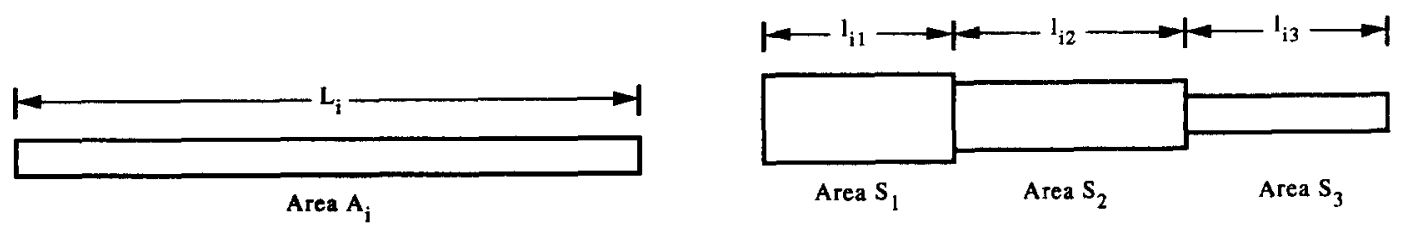

Fig. 4. Conventional member and segmental member. 


$$
\begin{aligned}
& \sigma_{j}^{L} \leqslant \sigma_{0 j}+\sum_{i=1}^{n}\left\{\frac{\partial \sigma_{0 j}}{\partial 1 / a_{i}}\left[\sum_{d=1}^{D} \frac{1}{s_{d}} \frac{l_{i d}}{l_{i}}-\frac{1}{a_{0 i}}\right]\right. \\
& +\frac{\partial \sigma_{0 j}}{\partial 1 / I_{i}}\left[\sum_{d=1}^{D} \frac{1}{I_{d}} \frac{l_{i d}}{l_{i}}-\frac{1}{I_{o i}}\right] \\
& \left.+\frac{\partial \sigma_{0 j}}{\partial \alpha_{i}}\left(\alpha_{i}-\alpha_{0 i}\right)\right\} \leqslant \sigma_{j}^{U} \\
& \alpha_{i}^{L} \leqslant \alpha_{i} \leqslant \alpha_{i}^{U} .
\end{aligned}
$$

The design variables of problem (22) are the lengths $l_{i d}$ of all segments of all the members and the fixity factors. The stress and displacement constraints are linear in both types of design variables. The discreteness requirement, eqn (14e), has been removed and absorbed into the problem by defining segments which have only the discrete sizes of set $S$. The additional constraints in problem (22) which do not appear in problem (14) are constraints like eqn (21), one for each member, which ensure length equivalence of conventional and segmental members, and the nonnegativity requirement for all segment lengths which are clearly necessary. Problem (22) is an LP problem which may be solved by any LP algorithm, and will yield what can be termed a segmental optimum design.

Several features of the segmental optimum design can be deduced. Firstly, its weight will be globally minimum for the explicit approximations on the displacements and stresses. This is a property of linear programming problems. This indeterminate frame will be solved in an iterative fashion and each of the sequence of LPs will be solved globally, although the sequence of global minima will not necessarily converge to a global minimum weight. This is an inherent feature of the iterative process and applies not just to segmental optimum design, but to all methods which solve minimum weight frame design problems iteratively. Secondly, the weight of the segmental optimum design, eqn (22), is a lower bound to the weight of the discrete optimum design of eqn (14).

\subsection{Achieving a discrete optimum design}

The discrete optimum design for a frame must have only one segment of discrete size per member. In the segmental optimum design most members will satisfy this requirement, but there will be a few multisegment members which do not. Some type of rounding operation is necessary. An obvious scheme is simply to round up all the multi-segment members, i.e. increase the size of all the smaller size segments in each member until they are of the same discrete cross-sectional area as the largest discrete size within the member. If all multi-segment members are treated in this way the result will be a feasible discrete design which may be the discrete optimum design and will in general be an upper bound to the discrete optimum design. Because only a few members of the frame are concerned in this rounding up operation the percentage weight increase should be small and the discrete design thus obtained, though perhaps not optimal, should have a weight only fractionally larger than the globally optimum discrete design. This rounding up can be done using the simplex table corresponding to the segmental optimum design. The round up operation is equivalent to setting the segment length variables of the smaller size segments within a multi-segment member to zero.

Having achieved a rounded-up discrete design from the segmental optimum design this is often as far as the method will go. Occasionally however, further refinement of the discrete design may be possible and it is comparatively easy to check this. The check consists of determining whether any complete members in the discrete design can be replaced by complete members of a smaller size without violating any constraints. The simplex table for the rounded up discrete design can be used for this. The slack variables of the displacement constraints will be in the basic set and must remain there with positive or zero values. The other basic variables are segment lengths, one per member with a value equal to the physical length of the member. The objective function coefficients will indicate several candidate segment length variables in the non-basic set which, if they entered the basis, would reduce the weight of the structure. Each candidate can be examined and pivoted into the basis provided that (a) it pivots a complete segment variable out of the basis, and (b) it does not violate the nonnegativity of any other basic variable. These extra checks and pivots will produce a new discrete design even closer to the optimum. It should be noted here that only the optimality of the segmental optimum design is guaranteed.

The rounding up and refinement processes are not rigorous and have no inherent guarantees of optimality. However, because the segmental optimum design is globally optimal and forms an almost discrete design, it is usually a close lower bound to the discrete optimum design. Because the number of membranes involved in the rounding is small, the rounded up segmental optimum design usually forms a close upper bound to the discrete optimum design. Any further refinements of this upper bound design will tighten further these already close bounds upon the discrete optimum design.

\subsection{Indeterminate structures}

Among the several ways in which the optimization strategy can be included in an iterative sequence of analysis-optimization cycles is to perform only a segmental optimum design in each iterative cycle until convergence of the sequence is almost complete. Rounding up and refinement are only added in at this late stage. This approach raises one small difficulty in that the structural analysis which separates each optimization must be carried out on a structure containing multi-segment members. For a multi-segment 


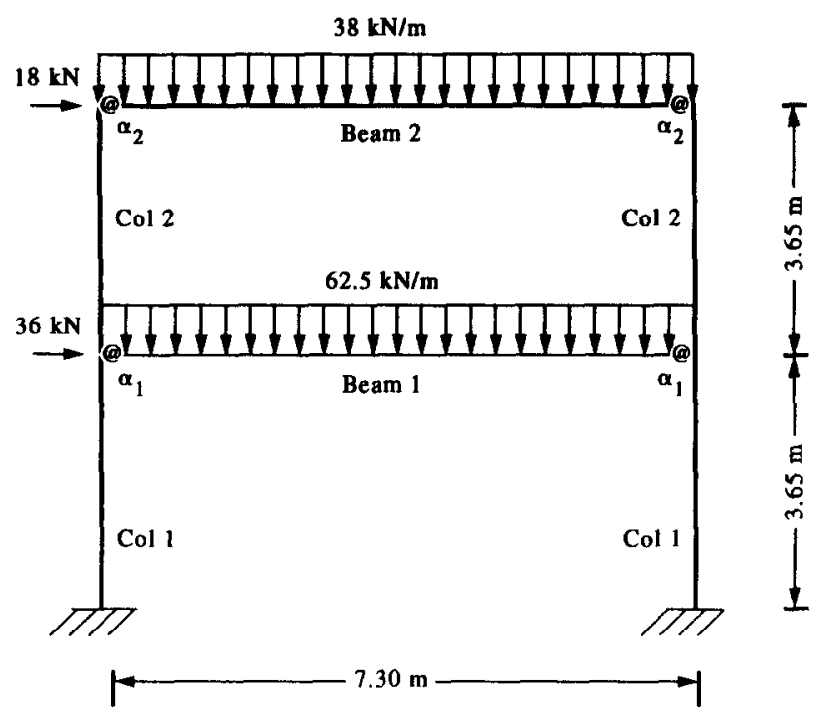

Fig. 5. Example 1: one-bay two-storey steel frame.

member with different lengths, material properties and areas for each segment, the simplest way of accommodating this within the stiffness matrix $\mathbf{K}$ is to calculate an equivalent stiffness value for the member rather than to attempt to write $K$ in terms of individual segments. The equivalent stiffness $k_{i}$ of a multi-segment axial force bar will be simply

$$
k_{i}=\Sigma_{1=1, n} \Sigma_{d=1, D} \frac{E_{p} s_{d}}{l_{i d l}} \text { for all } l_{i d l} \neq 0 \text {. }
$$

Using eqn (23) for axial forces and similarly for bending, all elements of $K$ corresponding to a segmental design can easily be calculated and the subsequent determination of the nodal displacement is straightforward.

\section{NUMERICAL APPLICATIONS}

\subsection{Example 1}

The one-bay, two-storey steel framework loaded as shown in Fig. $S$ has semi-rigid connections of equal fixity factors at the two ends of each of the beam members. The two column members at each storey level are prescribed to be the same size. The frame is to be designed in accordance with the strengthstability (stress) requirements, while, at the same time, ensuring that the top storey lateral sway at node 6 does not exceed $18.28 \mathrm{~mm}$ (i.e. $h / 400$ ). The cross-section area $a_{i}$ of each member is to be selected consistent with the requirement that all column and beam members are to have IPE sections. Young's modulus $E=206 \mathrm{kN} \mathrm{mm}^{-2}$ and the material density $\rho=7.85 \mathrm{t} \mathrm{m}^{-3}$. As such, the weight coefficient $w_{i}$ in eqn (5) for each column member of length $3.65 \mathrm{~m}$ is $w_{i}=28.65 \mathrm{t} \mathrm{m}^{-2}$, while that for each beam member of length $7.30 \mathrm{~m}$ is $w_{i}=57.305 \mathrm{t} \mathrm{m}^{-2}$. A quadratic connection cost with respect to $\alpha$ is adopted for cach beam-to-column joint.

A fixity factor $\alpha=0.9$ is used to define the initial rotational stiffness of each of the four semi-rigid connections for the framework. Upon applying the previously described iterative design optimization procedure, the optimal design of the frame having minimum member plus connection cost is found after two design stages. The optimal member IPE-sections are depicted in Fig. 6.
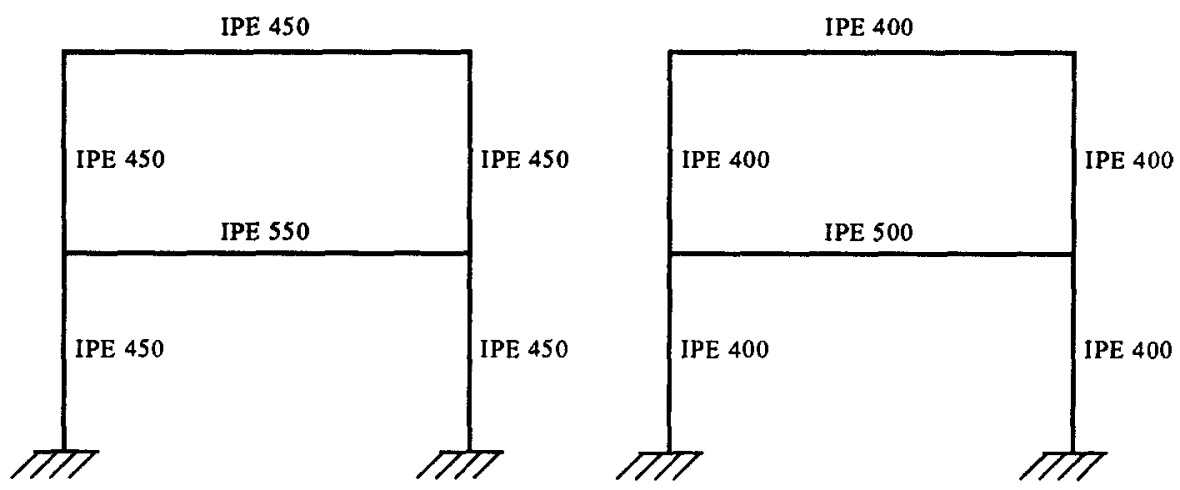

Fig. 6. Optimum solution fully rigid joints and optimum solution semi rigid joints. 


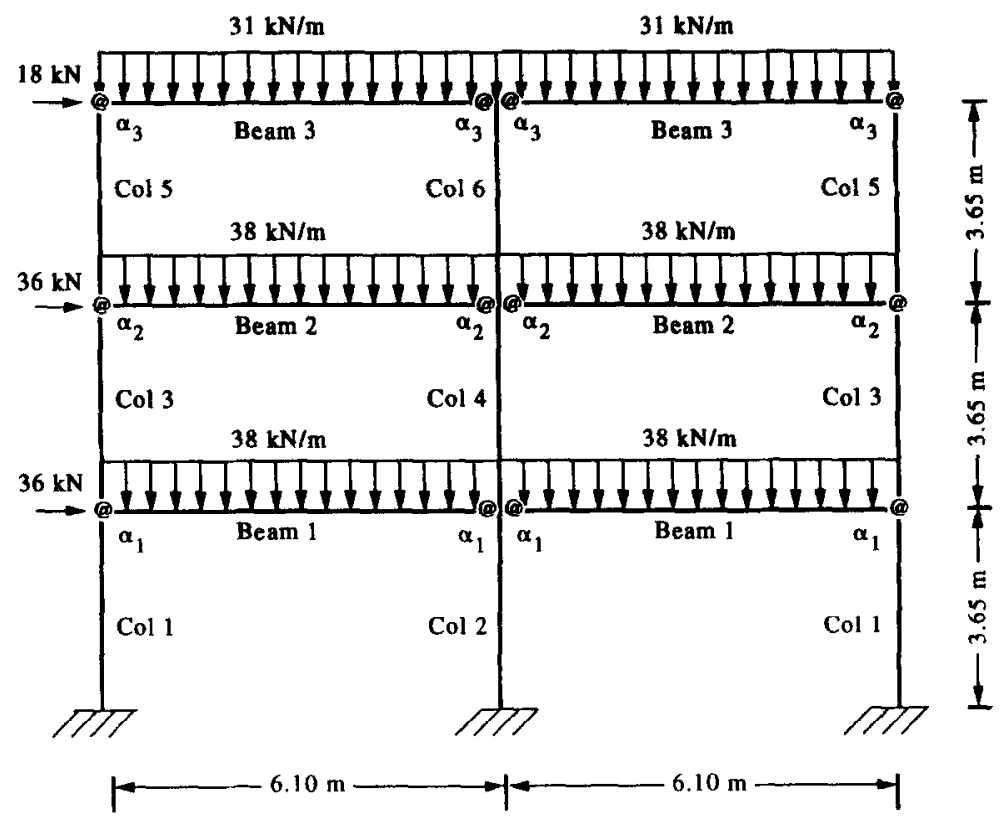

Fig. 7. Example 2: two-bay three-storey steel frame.

The optimal fixity factors of the semi-rigid connections are $\alpha_{1}=0.59\left(S_{1}=5.9 \times 10^{4} \mathrm{kNm} \mathrm{rad}^{-1}\right)$ for the lower-storey beam and $\alpha_{2}=0.66\left(S_{2}=3.8 \times\right.$ $10^{4} \mathrm{kNm} \mathrm{rad}^{-1}$ ) for the upper-storey beam. Also shown in Fig. 6 is the optimum solution for the case when the connections are assumed to be fully rigid. Interestingly, the design that accounts for semi-rigid connections weighs $14 \%$ less than the design found when only fully-rigid connections are considered.

\subsection{Example 2}

The two-bay, three-storey steel framework loaded as shown in Fig. 7 has semi-rigid connections at the ends of the beam members. Connection fixity factors are specified to be the same for all semi-rigid connections at each storey level. External column members at each storey level are prescribed to have the same IPE section, as are beam members at each storey level. The frame is to be designed in accordance with the strength-stability (stress) requirements, while ensuring that the top-storey lateral sway does not exceed $27.38 \mathrm{~mm}$.

The material density $\rho=7.85 \mathrm{t} \mathrm{m}^{-3}$ and, as such, the weight coefficient for each column member is $w_{i}=28.65 \mathrm{t} \mathrm{m}^{-2}$, while that for each member is $w_{i}=47.885 \mathrm{~lm}^{-2}$. As for Example 1, the connection cost is also assumed quadratic in $\alpha$.

A fixity factor $\alpha=0.9$ is adopted to define the initial rotational stiffness of each of the semi-rigid connections for the framework. Upon applying the iterative design optimization procedure, the optimal design of the frame having a minimum member plus connection cost is found after four design stages. The optimal momber IPE-sections are depicted in Fig. 8.

The optimal rotational stiffness of the semi-rigid connections area $\alpha_{1}=0.6\left(S_{1}=2.5 \times 10^{4} \mathrm{kN} \mathrm{m} \mathrm{rad}^{-1}\right)$ for the first storey, $\alpha_{2}=0.575\left(S_{2}=2.2 \times 10^{4} \mathrm{kN} \mathrm{m}\right.$ $\left.\mathrm{rad}^{-1}\right)$ for the second storey and $\alpha_{3}=0.55\left(S_{3}=1.5\right.$ $\times 10^{4} \mathrm{kN} \mathrm{m} \mathrm{rad}^{-1}$ ) for the third storey. Also shown in Fig. 8 is the optimum solution when all connections
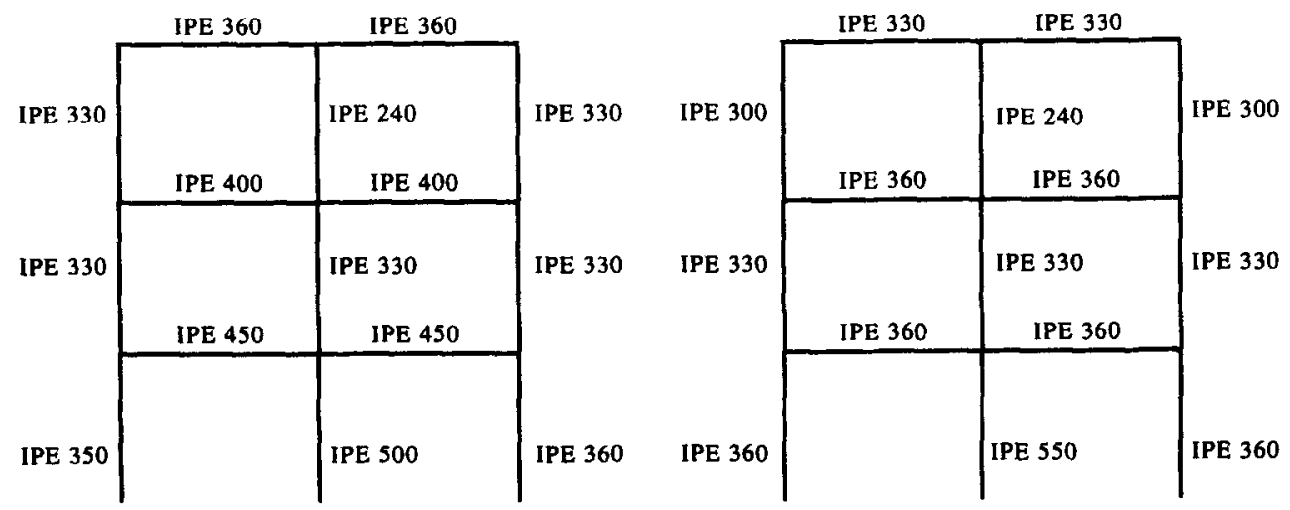

Fig. 8. Optimum solution fully rigid joints and optimum solution semi rigid joints. 
are assumed to be fully rigid. As for Example 1, the fully-rigid connection design weighs $12 \%$ more than the design found when the influence of semi-rigid connections is accounted for.

\section{CONCLUDING REMARKS}

The described optimization procedure provides an effective means to account for the cost of both members and connections in the design of steel building frameworks. In fact, accounting for the actual semirigid behaviour of connections results in designs that are less costly than when, as is usually done, the connections are idealized as being fully rigid. This is because semi-rigid connections allow for a redistribution of internal member forces (shear, moment, etc.) that results in a more economical use of material to resist the applied loads. This is an important result because it implies that accounting for the actual semi-rigid behaviour of connections in the design of steel frameworks is both realistic and economical.
Acknowledgement-The author wishes to thank the financial support given by JNICT (Junta Nacional de Investigação Científica e Tecnológica), Project STRDA/C/TPR/576/92.

\section{REFERENCES}

1. D. Anderson, F. Bijlaard, D. A. Nethercot and R. Zandonini, Analysis and design of steel frames with semi-rigid connections. IABSE Surv, 4, 61-78 (1987).

2. D. Anderson and K. Kavianpur, Analysis of steel frames with semi-rigid connections. Struct. Engng Rev. 3, 79-87 (1991).

3. R. Cunningham, Some aspects of semi-rigid connections in structural steelwork. Struct. Engng 68, 85-92 (1990).

4. D. E. Grierson and L. Xu, Optimal design of steel frameworks accounting for semi-rigid connections. In: Optimization of Large Structural Systems (Edited by G. I. Rozvany). Kluwer, Dordrecht (1992).

5. G. R. Monforton and T. S. Wu, Matrix analysis of semi-rigidly connected steel frames. ASCE struct. Div'. 89, 13-42 (1963).

6. L. M. C. Simôes, Reliability of portal frames with discrete design variables. Struct. Optim. 5, 76-83 (1992).

7. A. B. Templeman and D. F. Yates, A segmental method for the discrete optimum design of structures. Engng Optim. 6, 145-155 (1983). 